\title{
Distribuição espacial e sazonal de anfíbios em quatro poças na região serrana do Espírito Santo, sudeste do Brasil: influência de corredores florestais
}

\author{
Rodrigo Barbosa Ferreira ${ }^{1,2,4}$, Roberto de Barros Dantas ${ }^{1} \&$ João Filipe Riva Tonini ${ }^{3}$ \\ 1. Museu de Biologia Mello Leitão, Av. José Ruschi, n 4, 29650-000, Santa Teresa, Espírito Santo, Brasil. (rodrigoecologia@yahoo.com.br; dantasbio@hotmail.com) \\ 2. Department of Wildland Resources and Ecology Center, Utah State University, Logan, Utah 84322-5230, USA. \\ 3. Department of Biology, University of Richmond, Richmond, VA 23173, USA. (jfrtonini@gmail.com) \\ 4. Autor para correspondência.
}

\begin{abstract}
Spatial and seasonal distribution of amphibians in four ponds at mountainous region in southeastern of Brazil: influence of forest corridor. This study evaluated the composition of anuran assemblages, and also the spatial and temporal distribution of those species in four permanent ponds. Two of these ponds are connected to Atlantic rainforest fragments by forest corridor and the other two are surrounded by Eucalyptus plantation and human settlement. Twenty-two species from five families were recorded from December 2003 to November 2004. Most species and individuals collected belong to Hylidae, which presented pronounced spatial overlap. Vegetation under $0.60 \mathrm{~m}$ was the most used calling site. Although not statistically supported, species richness and abundance were higher in rainy than dry months. Ponds with corridors had higher species richness and fourteen species were exclusively found on these ponds. The community structure of anurans in permanent ponds at this fragmented landscape is apparently dictated by the presence or absence of forest corridors connecting ponds to forest fragments.
\end{abstract}

KEYWORDS. Anurans, richness, spatial-temporal distribution, habitat split.

RESUMO. Este estudo avaliou a composição da anurofauna, bem como a distribuição espacial e temporal das espécies em quatro poças permanentes em uma região montanhosa do Espírito Santo, sudeste do Brasil. Duas dessas poças são conectadas a fragmentos florestais por corredores florestais e as outras duas poças são circundadas por plantação de eucalipto e habitação humana. Foram registradas 22 espécies, incluídas em cinco famílias, entre dezembro de 2003 a novembro de 2004. Hylidae foi a mais rica e abundante, apresentando elevada sobreposição espacial. Nove espécies foram coletadas em vegetação abaixo de $0,60 \mathrm{~m}$, sendo o sítio de vocalização mais utilizado. Riqueza de espécies e abundância foram maiores em meses chuvosos. Poças com corredores abrigaram maior riqueza de espécies. Adicionalmente quatorze espécies foram exclusivamente encontradas nessas poças. A estrutura da comunidade de anuros em poças permanentes nessa paisagem fragmentada é aparentemente determinada pela presença ou ausência de corredores florestais conectando fragmentos em topos de morro às poças d'água ocorrentes ao longo dos vales.

PALAVRAS-CHAVE. Anuros, riqueza de espécies, distribuição espacial-temporal, desconexão do habitat.

Estudos que avaliam a distribuição temporal e espacial dos organismos são fundamentais para o entendimento dos processos de inter-relação com o ambiente. Coexistência de anuros durante atividade reprodutiva envolve a utilização distinta de habitats, sítios de vocalização, do período do ano e diferenças no canto (Bernarde \& Anjos, 1999; Bertoluci \& Rodrigues, 2002; Prado \& Pombal Jr, 2005; Campos \& VAZ-SILVA, 2010). Em termos temporais, precipitação tem sido o principal indicador climático de aumento da atividade reprodutiva em anuros para regiões tropicais (Duellman, 1999).

Diferentemente dos estados da Região Sudeste, o Espírito Santo apenas recentemente começou receber pesquisas focadas no entendimento dos fatores que atuam na estruturação das comunidades de anuros (ver Prado \& Pombal JR, 2005; Teixeira et al., 2008). Estudos com esse foco podem ser favorecidos após o substancial aumento no número de inventários herpetofaunísticos conduzidos recentemente no estado (ver Ramos \& GaSPARInI, 2004; RöDDER et al., 2007; TeIXeIra et al., 2008; Ferreira \& Mendes, 2010; Ferreira et al., 2010; Tonini et al., 2010; Silva-SoAres et al., 2011; Tonini et al., 2011; MonTEsinos et al., 2012), o que culminou na elaboração da lista estadual de anuros (Almeida et al., 2011).
A região serrana do Espírito Santo mesmo sendo destacada como um "hotspot" de biodiversidade (RöDDER et al., 2007), é alvo de uma crescente expansão agro-florestal e criação de condomínios de chácaras, o que tem gerado intensa modificação na paisagem original. Essa interferência antrópica pode resultar em separação espacial entre fragmentos florestais e habitats reprodutivos, prejudicando a migração de anfíbios adultos para ambientes reprodutivos e de juvenis para ambientes terrestres, fenômeno conhecido como desconexão do habitat (BECKER et al., 2010). Dessa forma, torna-se vital entender o papel de corredores florestais nessa região fragmentada, pois os mesmos potencialmente exercem forte influência na estrutura da comunidade de anuros.

O objetivo do presente estudo foi avaliar a dinâmica espacial e sazonal da comunidade de anuros em uma paisagem fragmentada sobre fortes pressões antrópicas na região serrana do Espírito Santo. Para isso investigamos i) se a composição de espécies altera-se ao longo de quatro poças permanentes; ii) se a atividade reprodutiva das espécies está correlacionada a variáveis climáticas; iii) e os potenciais efeitos da desconexão do habitat sobre a comunidade de anuros. 


\section{MATERIAL E MÉTODOS}

O trabalho foi realizado no Sítio Amarildo Uliana, Sítio Jaime Stein, e no Condomínio de chácaras Recanto das Flores, distrito de Alto Nova Almeida (20²3’29”S, 4050’42”W; altitude $916 \mathrm{~m})$, município de Marechal Floriano, estado do Espírito Santo (Fig. 1). De acordo com KöPPEN (1936), o clima da região é do tipo $\mathrm{Cwb}$, tropical de altitude, temperado húmido, com inverno seco e verão temperado. As temperaturas oscilam entre $12^{\circ} \mathrm{C}$ e $20^{\circ} \mathrm{C}$, conforme altitude; o índice pluviométrico médio é de $1.500 \mathrm{~mm} / \mathrm{ano}$ (FeitozA, 1986). A vegetação original era Floresta Ombrófila Densa que atualmente distribui-se principalmente no topo dos morros. As florestas que ocorriam ao longo dos vales da região foram em sua maioria amplamente alterada para estabelecimento de propriedades particulares, sobretudo agrícolas. Atualmente há uma crescente substituição de culturas agrícolas tradicionais e desmatamento de matas em regeneração para plantio de eucalipto.

O estudo foi realizado em quatro poças d'água permanentes, formadas a partir do represamento de um córrego afluente do rio Fundo. Expedições mensais ocorreram de dezembro de 2003 a novembro de 2004, com um dia de duração, iniciadas no início do crepúsculo $(18: 00 \mathrm{~h})$ e término 0:00 $\mathrm{h}$. Os métodos de registro foram visualização direta das espécies e reconhecimento das vocalizações. A temporada de vocalização de cada espécie foi determinada com base no registro de machos adultos vocalizando.

As poças estudadas foram: Poça 1) área de 233 $\mathrm{m}^{2}$ com gramíneas nas margens e consideravelmente antropizada, cercada em sua maioria por cultivo de

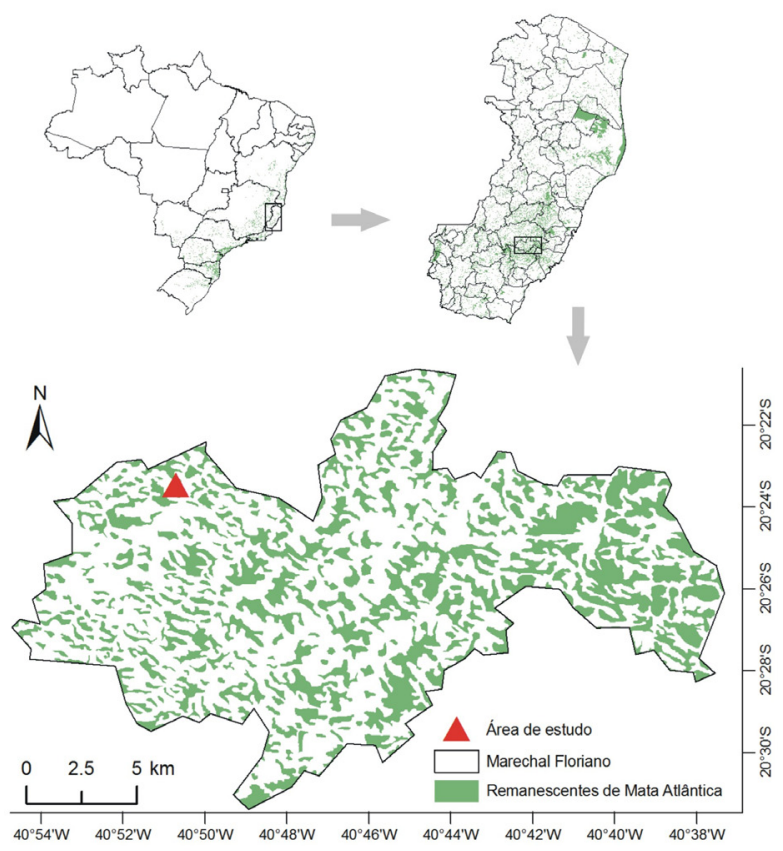

Fig. 1. Localização da área de estudo, Marechal Floriano, região serrana do Espírito Santo, sudeste do Brasil. eucalipto e por habitações; Poça 2) $324 \mathrm{~m}^{2}$ de área, com vegetação e entorno semelhantes à Poça 1; Poça 3) área de $61 \mathrm{~m}^{2}$, circundada em sua maioria por arbustos que conectam esse corpo d'água a um remanescente de mata secundária em topo de morro; vegetação aquática flutuante, principalmente Salvinia sp., cobre uma significativa porção da mesma; Poça 4) área de $320 \mathrm{~m}^{2}$ circundada em sua maior parte por cultura agrícola; possui também uma faixa de vegetação de 24 metros de largura que a conecta a um fragmento de mata primária.

Os sítios de vocalização onde havia machos vocalizando foram registrados e divididos da seguinte maneira: A) ao nível da superfície da água, com o corpo parcialmente submerso ou flutuando nos corpos d'água; B) no chão sobre o solo seco; C) ao nível da superfície da água, sobre folhas de plantas aquáticas; D1) aderido às gramíneas emergentes ou marginais aos corpos d'água; D2) suprimindo gramíneas marginais aos corpos d'água; E1) na vegetação arbustiva ou arbórea abaixo de $0,60 \mathrm{~m}$; E2) na vegetação arbustiva ou arbórea acima de $0,60 \mathrm{~m}$ até $1,5 \mathrm{~m}$; E3) na vegetação arbustiva ou arbórea acima $1,50 \mathrm{~m}$; F) folhas de taboa (Thypha sp.); e G) no solo marginal formando ninhos (poças construídas).

Quanto à ocorrência temporal, as espécies foram classificadas em: 1) constante, para espécies registradas vocalizando em, pelo menos, dez meses do ano; 2 ) semiconstante, para espécies registradas vocalizando por um período de 3 a 9 meses; e 3) ocasional, para espécies registradas vocalizando em um período inferior a 3 meses (Prado \& Pombal JR, 2005).

Os dados abióticos (pluviosidade, temperaturas máxima e mínima) foram obtidos na Estação Agrometereológica do Incaper/INMET (INCAPER, 2006), localizada a $31 \mathrm{~km}$ da área de estudo, no Município de Venda Nova do Imigrante.

Para avaliar a correlação entre os dados bióticos e abióticos foi utilizado teste de correlação de Pearson (ZAR, 1999). A curva de acumulação de espécies foi elaborada para simular o número de espécies observadas de acordo com o número de visitas a campo. A riqueza esperada foi estimada pelo índice Chao 2 (intervalo de confiança de 95\%), o qual tem sido usado em inventários de curta duração (CHAZDON et al., 1998).

Foi gerada uma matriz de similaridade, utilizando o índice de Jaccard, para os dados de presença/ ausência de anuros: i) ao longo das poças e ii) sítios de vocalização. As poças e os sítios de vocalização foram comparados através da Análise de Agrupamento (ou "cluster"; Clarke, 1993), pelo método de UPGMA com união de grupos, e os agrupamentos foram testados pelo Similarity Profile (SIMPROF) utilizando 500 replicações, relações significativas com $p<0,05$. Todas análises foram realizadas no programa Primer-6 (CLARKE \& GORLEY, 2006). 


\section{RESULTADOS}

Ao longo das quatro poças permanentes registramos um total de 287 indivíduos pertencentes a 22 espécies (Tab. I). Hylidae apresentou a maior riqueza de espécies $(\mathrm{N}=17)$, seguida por Leptodactylidae $(\mathrm{N}=2)$, Bufonidae, Leiuperidae e Cycloramphidae $(\mathrm{N}=1)$. Um indivíduo de Scinax não teve sua identificação confirmada.

A curva do coletor apresentou uma relação direta com o número de coletas, mas não atingiu uma assíntota (Fig. 2). Entretanto o estimador de riqueza Chao 2 estimou 22 espécies, o que corresponde à riqueza observada após considerarmos os dois registros feitos em coletas adicionais (L. fuscus e T. miliaris).

Dendropsophus branneri, $L$. cf. latrans e $S$. alter estiveram presentes em todas as poças, sendo consideradas espécies generalistas quanto ao habitat (Tab. I). A análise de agrupamento revelou que a comunidade de anuros, quanto à ocupação das poças, está dividida em dois agrupamentos: i) espécies que ocupam poças ausentes de corredor florestal conectando-

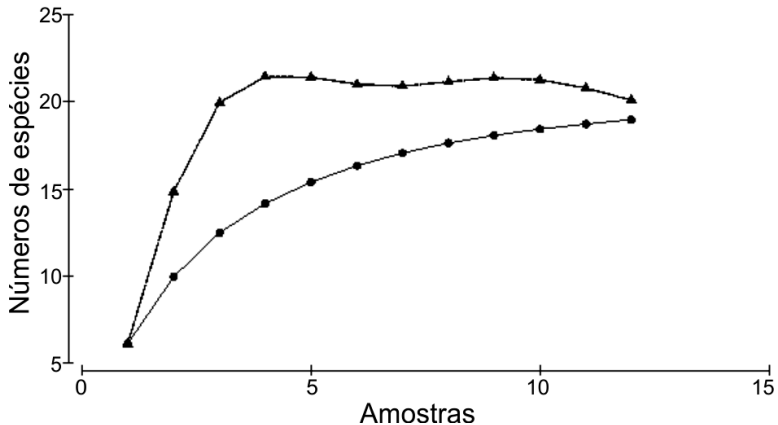

Fig. 2. Curva de acumulação de espécies (círculo) e riqueza estimada (triângulo) de acordo com o índice Chao 2, calculada com os dados obtidos entre dezembro de 2003 a novembro de 2004.

as aos fragmentos florestais (1 e 2, Fig. 3); ii) espécies que ocupam poças que possuem corredores florestais conectando-as aos fragmentos florestais (3 e 4, Fig. 3). Poças 3 e 4 apresentaram riqueza de espécies superior às anteriores. Adicionalmente poças com corredores (3 e 4) abrigaram quatorze espécies exclusivas, enquanto apenas três espécies foram registradas exclusivamente ocupando poças ausentes de corredores ( 1 e 2, Tab. I).

Tab. I. Distribuição espacial das 22 espécies de anuros registradas em quatro poças analisadas na região serrana do Espírito Santo [*, espécies registradas em coletas adicionais, após o término do período de estudo. Sítios de vocalização: A) ao nível da superfície da água com o corpo parcialmente submerso ou flutuando nos corpos d'água; B) no chão sobre o solo seco; C) ao nível da superfície da água, sobre folhas de plantas aquáticas; D1) aderido às gramíneas emergentes ou marginais aos corpos d'água; D2) suprimindo gramíneas marginais aos corpos d'água; E1) na vegetação arbustiva ou arbórea abaixo de $0,60 \mathrm{~m}$; E2) na vegetação arbustiva ou arbórea acima de $0,60 \mathrm{~m}$ até $1,5 \mathrm{~m}$; E3) na vegetação arbustiva ou arbórea acima 1,50 m; F) folhas de taboa (Thypha sp.); G) no solo marginal formando ninhos (poças construídas)].

\begin{tabular}{|c|c|c|c|c|c|}
\hline \multirow{2}{*}{ Espécies } & \multicolumn{4}{|c|}{ Poças } & \multirow{2}{*}{ Sítios de vocalização } \\
\hline & 1 & 2 & 3 & 4 & \\
\hline \multicolumn{6}{|l|}{ Bufonidae } \\
\hline Rhinella crucifer (Wied-Neuwied, 1821) & $\mathrm{X}$ & $\mathrm{X}$ & & & $\mathrm{A}, \mathrm{B}, \mathrm{D} 2$ \\
\hline \multicolumn{6}{|l|}{ Hylidae } \\
\hline Bokermannohyla caramaschii (Napoli, 2005) & & & & $\mathrm{X}$ & E2 \\
\hline Dendropsophus branneri (Cochran, 1948) & $\mathrm{X}$ & $\mathrm{X}$ & $\mathrm{X}$ & $\mathrm{X}$ & $\mathrm{C}, \mathrm{D} 1, \mathrm{E} 1, \mathrm{~F}$ \\
\hline Dendropsophus decipiens (A. Lutz, 1925) & & $\mathrm{X}$ & $\mathrm{X}$ & $\mathrm{X}$ & $\mathrm{C}, \mathrm{D} 1$ \\
\hline Dendropsophus elegans (Wied-Neuwied, 1824) & & & $\mathrm{X}$ & $\mathrm{X}$ & $\mathrm{D} 1, \mathrm{E} 1, \mathrm{E} 2, \mathrm{~F}$ \\
\hline Dendropsophus minutus (Peters, 1872) & & & $\mathrm{X}$ & $\mathrm{X}$ & $\mathrm{C}, \mathrm{D} 1, \mathrm{E} 1, \mathrm{~F}$ \\
\hline Hypsiboas albomarginatus (Spix, 1824) & & & $\mathrm{X}$ & $\mathrm{X}$ & $\mathrm{E} 1, \mathrm{E} 2$ \\
\hline Hypsiboas albopunctatus (Spix, 1824) & $\mathrm{X}$ & $\mathrm{X}$ & & $\mathrm{X}$ & D1, E1 \\
\hline Hypsiboas crepitans (Wied-Neuwied, 1824) & $\mathrm{X}$ & $\mathrm{X}$ & & & $\mathrm{B}, \mathrm{G}$ \\
\hline Hypsiboas faber (Wied-Neuwied, 1821) & & & $\mathrm{X}$ & $\mathrm{X}$ & $\mathrm{B}, \mathrm{E} 2, \mathrm{G}$ \\
\hline Hypsiboas pardalis (Spix, 1824) & $\mathrm{X}$ & $\mathrm{X}$ & & & $\mathrm{B}, \mathrm{E} 2, \mathrm{G}$ \\
\hline Hypsiboas polytaenius (Cope, 1870 ) & & & $\mathrm{X}$ & & \\
\hline Hypsiboas semilineatus (Spix, 1824) & & & $\mathrm{X}$ & $\mathrm{X}$ & $\mathrm{E} 2, \mathrm{E} 3$ \\
\hline Phyllomedusa rohdei Mertens, 1926 & & & $\mathrm{X}$ & & $\mathrm{E} 2, \mathrm{E} 3$ \\
\hline Scinax alter (B. Lutz, 1973) & $\mathrm{X}$ & $\mathrm{X}$ & $\mathrm{X}$ & $\mathrm{X}$ & $\mathrm{B}, \mathrm{D} 1, \mathrm{E} 1$ \\
\hline Scinax hayii (Barbour, 1909) & & & & $\mathrm{X}$ & E1 \\
\hline Scinax x-signatus (Spix, 1824) & & & & $\mathrm{X}$ & E1 \\
\hline Scinax sp. & & & & $\mathrm{X}$ & E1 \\
\hline \multicolumn{6}{|l|}{ Leiuperidae } \\
\hline Physalaemus cuvieri Fitzinger, 1826 & & & & $\mathrm{X}$ & A \\
\hline \multicolumn{6}{|l|}{ Leptodactylidae } \\
\hline Leptodactylus cf. latrans (Steffen, 1815) & $\mathrm{X}$ & $\mathrm{X}$ & $\mathrm{X}$ & $\mathrm{X}$ & A, D2 \\
\hline *Leptodactylus fuscus (Schneider, 1799) & & & $\mathrm{X}$ & & \\
\hline \multicolumn{6}{|l|}{ Thoropidae } \\
\hline *Thoropa miliaris (Spix, 1824) & & & $\mathrm{X}$ & & \\
\hline Total de espécies & 7 & 8 & 13 & 15 & \\
\hline
\end{tabular}




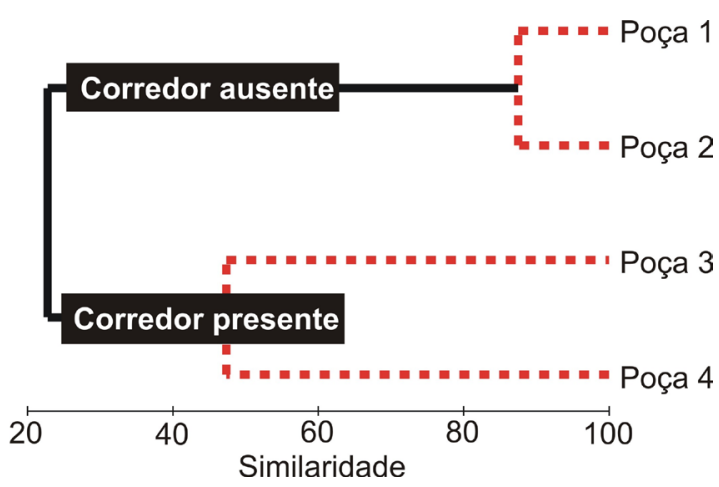

Fig. 3. Análise de agrupamento demonstrando a similaridade entre as quatro poças amostradas em relação à composição das espécies. Agrupamentos em preto apresentaram $\mathrm{p}<0,05$ pelo SIMPROF.

O sítio de vocalização mais utilizado foi vegetação arbustiva ou arbórea abaixo de $0,60 \mathrm{~m}$ (E1). Nesse local também ocorreu três das cinco espécies que foram exclusivas a um único sítio de vocalização (Tab. I). Contrariamente, vegetação arbustiva ou arbórea acima de $1,50 \mathrm{~m}$ (E3) foi o menos utilizado, sendo $P$. rohdei e $H$. semilineatus específicas a esse substrato. Dendropsophus branneri, D. elegans e D. minutus foram as mais generalistas, ocupando quatro tipos diferentes de sítios de vocalização. O "cluster" demonstrou a formação de três grupos distintos (Fig. 4): i) formado por espécies que vocalizam no solo (B), espécies arborícolas

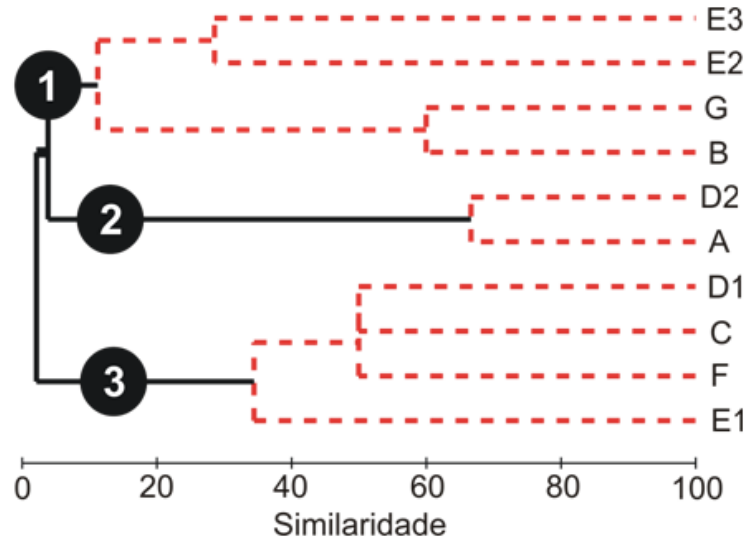

Fig. 4. Análise de agrupamento demonstrando a similaridade dos sítios de vocalização utilizados quanto à composição das espécies. Agrupamentos em preto apresentaram $\mathrm{p}<0,05$ pelo SIMPROF.

que vocalizam acima de $0,60 \mathrm{~m}$ de altura (E2, E3) e espécies que vocalizam em ninhos construídos no solo nas margens dos corpos d'água (G); ii) composto por espécies que vocalizam sobre gramíneas nas margens dos corpos d'água (D2) e ao nível da superfície da água (A), e iii) formado por espécies arborícolas que vocalizam abaixo de $0,60 \mathrm{~m}$ de altura, em gramíneas ao redor e no interior dos corpos d'água (D1), na superfície da água sobre folhas de plantas aquáticas (C) e sobre folhas de taboa (F).

Tab. II. Abundância mensal das espécies entre dezembro de 2003 a novembro de 2004 na região serrana do Espírito Santo [Abundância: tracejado horizontal < 5; grade $\geq 5$ e $\leq 10$; tracejado vertical >10 indivíduos. Duração da temporada de vocalização (sazonalidade): S, semi-constante; O, ocasional]. As espécies registradas em coletas adicionais (L. fuscus e T. miliaris) foram removidas dessa tabela.

\begin{tabular}{|c|c|c|c|c|c|c|c|c|c|c|c|c|c|}
\hline \multirow[t]{2}{*}{ Espécies } & \multicolumn{12}{|c|}{ Meses } & \multirow[t]{2}{*}{ Saz. } \\
\hline & Dez & Jan & Fev & Mar & Abr & Mai & Jun & Jul & Ago & Set & Out & Nov & \\
\hline \multicolumn{14}{|l|}{ Bufonidae } \\
\hline R. crucifer & 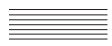 & & & & & & & & 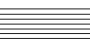 & 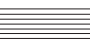 & & & $\mathrm{S}$ \\
\hline \multicolumn{14}{|l|}{ Hylidae } \\
\hline B. caramaschii & & & & & & & & 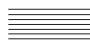 & $\bar{~}$ & & & & $\mathrm{~S}$ \\
\hline D. branneri & \|\||||| $\mid$ & 地地 & $\||\|\mid\|$ & $\overline{\underline{\underline{\underline{\underline{\underline{n a n}}}}}}$ & & & & 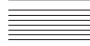 & & & & & $\mathrm{S}$ \\
\hline D. decipiens & & & & & & & & & & 宸 & & & $\mathrm{S}$ \\
\hline D. elegans & & & & & & & & & & & & & $\mathrm{S}$ \\
\hline D. minutus & & \# & & $\overline{\overline{\overline{\underline{\underline{\underline{y}}}}}}$ & & & & & & 淁 & & & $\mathrm{S}$ \\
\hline H. albomarginatus & & 事 & & & & & & & & 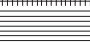 & & $\overline{\overline{\underline{\underline{\underline{\underline{y^{\prime}}}}}}}$ & $\mathrm{S}$ \\
\hline H. albopunctatus & 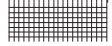 & & & 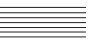 & & & & 産言 & & $\bar{E}$ & & & $\mathrm{~S}$ \\
\hline H. crepitans & 跨高 & & & & & & & & 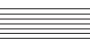 & & & & $\mathrm{O}$ \\
\hline H. faber & 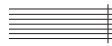 & 地 & 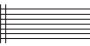 & & & & & & & 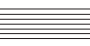 & & $\overline{\overline{\underline{\underline{\underline{\underline{y^{\prime}}}}}}}$ & $\mathrm{S}$ \\
\hline H. pardalis & 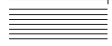 & & & & & & & & 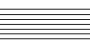 & & & & $\mathrm{O}$ \\
\hline H. polytaenius & & & & & & & & 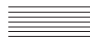 & & & & $\overline{\overline{\underline{\underline{\underline{\underline{\underline{v^{\prime}}}}}}}}$ & $\mathrm{S}$ \\
\hline H. semilineatus & $\overline{\overline{\underline{\underline{\underline{\underline{\underline{y}}}}}}}$ & & & 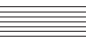 & & & & & & & & & $\mathrm{O}$ \\
\hline P. rohdei & & & & & & & & & & & & & $\mathrm{S}$ \\
\hline S. alter & 增 & \|\|$\|$ & \|\|\|\|$\|$ & 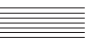 & & & & 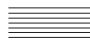 & 賏|||||||||||| & \#地地 & \|\||||||| & $|\||\|||||||||| \mid$ & $\mathrm{S}$ \\
\hline S. hayii & & & & & & & & & 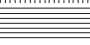 & & & & $\mathrm{O}$ \\
\hline Scynax sp. & & & & & & & & & & 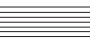 & & & $\mathrm{O}$ \\
\hline S. x-signatus & & & & & & & & & & & & & $\mathrm{O}$ \\
\hline \multicolumn{14}{|l|}{ Leiuperidae } \\
\hline P. cuvieri & & 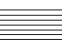 & & & & & & 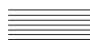 & & & & & $\mathrm{O}$ \\
\hline \multicolumn{14}{|l|}{ Leptodactylidae } \\
\hline L. cf. latrans & $\overline{\overline{\underline{\underline{\underline{\underline{\underline{y}}}}}}}$ & & 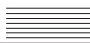 & $\overline{\overline{\overline{\underline{\underline{\underline{y}}}}}}$ & & & & & & & 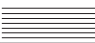 & & $\mathrm{S}$ \\
\hline Abundância & 46 & 43 & 35 & 17 & 0 & 0 & 0 & 12 & 33 & 37 & 35 & 27 & \\
\hline Total de espécies & 13 & 10 & 8 & 8 & 0 & 0 & 0 & 6 & 9 & 11 & 5 & 7 & \\
\hline
\end{tabular}


As espécies foram reunidas em dois grupos quanto à ocorrência temporal (Tab. II). Sete espécies adotaram o padrão de vocalização ocasional (menor do que três meses), enquanto que o restante das espécies (13) apresentaram padrão semi-constante. Nenhuma espécie apresentou padrão do tipo constante, pois machos não foram visualizados vocalizando durante abril, maio e junho. Os meses mais chuvosos tiveram maior riqueza de espécies e maior abundância de indivíduos (Tab. II). No entanto, não houve correlação significativa entre os valores de pluviosidade, e temperaturas máximas e mínimas, com a riqueza de anuros em vocalização (Tab. III).

Tab. III. Correlação de Pearson entre parâmetros abióticos e riqueza de espécies de anuros na região serrana do Espírito Santo.

\begin{tabular}{lcc}
\hline Parâmetros abióticos & $\mathrm{R}$ & $\mathrm{p}$ \\
\hline Temperatura mínima $\left({ }^{\circ} \mathrm{C}\right)$ & 0,41 & 0,26 \\
Temperatura máxima $\left({ }^{\circ} \mathrm{C}\right)$ & 0,40 & 0,28 \\
Pluviosidade $(\mathrm{mm})$ & 0,11 & 0,77 \\
\hline
\end{tabular}

\section{DISCUSSÃO}

A composição da anurofauna registrada no local de estudo corresponde a $16 \%$ das espécies listadas para o Espírito Santo (Almeida et al., 2011) e assemelha-se com outras comunidades de anuros amostradas na região montanhosa capixaba (PP em RöDDER et al., 2007; Montesinos et al., 2012). Nenhuma espécie registrada está incluída na lista de anfíbios ameaçados do Espírito Santo (GASPARINI et al., 2007). Em fato, a maioria delas possui ampla distribuição na Mata Atlântica, ocorrendo também em outros biomas (D. minutus, $H$. albomarginatus, $H$. albopunctatus, $H$. crepitans, $L$. cf. latrans, P. cuvieri e $S$. x-signatus). Houve também registros de espécies típicas de regiões montanhosas da Mata Atlântica (H. polytaenius e B. caramaschii). Hypsiboas polytaenius e $P$. rohdei foram registradas pela primeira vez para o Espírito Santo ao longo das campanhas desse estudo (ver FerReira \& VRCibradic, 2005; VRCIBRADIC et al., 2005).

Dendropsophus branneri $\mathrm{e}$ S. alter são consideradas oportunistas por serem capazes de colonizar rapidamente e aumentar a atividade reprodutiva em ambientes modificados (ver BABBITT \& TANNER, 2000; IZECKSON \& Carvalho-e-Silva, 2001). Corroborando com essa afirmação, essas espécies foram as mais abundantes tanto em áreas montanhosas (presente estudo), quanto em uma região de restinga do Espírito Santo (TeixeIRa et al., 2008). Outro exemplo de oportunismo evidenciado no presente estudo é o fato dessas espécies ocorrerem em todas as quatro poças e ocuparem uma grande variedade de sítios de vocalização.

Além de fatores ecológicos, tamanho e adaptações morfológicas estão também envolvidos na escolha dos sítios de vocalização (HodL, 1977). As espécies exclusivas a cada um dos três grupos foram as responsáveis pela distinção espacial identificada no "cluster". O maior tamanho corpóreo das espécies exclusivas do grupo 1 (CRC $=38$ a $100 \mathrm{~mm}$; NAPOLI, 2005; Wogel et al., 2005; CASAL \& Juncé, 2008; Forlani et al., 2010) em relação às espécies dos outros grupos parece ser a principal característica pela formação desse agrupamento. A presença de $R$. crucifer no grupo 1, que é formado em sua maioria por espécies que vocalizam sobre a vegetação, deve-se principalmente a $H$. faber $(\mathrm{CRC}=90$ a $100 \mathrm{~mm})$. Indivíduos dessa espécie vocalizam em árvores, porém constroem seus ninhos no solo (Izeckson \& CARVAlHo-e-Silva, 2001). As espécies com discos adesivos ocorreram exclusivamente nos grupos 1 e 3, havendo grande compartilhamento de espécies dentre estes. No grupo 2, as espécies exclusivas vocalizam ao nível da superfície da água, ambiente capaz de sustentar tanto espécies pequenas quanto de maior tamanho, variando de $P$. cuvieri $(\mathrm{CRC}=31$ mm; ForLANi et al., 2010) a L. latrans (CRC $=92$ a 108 mm; Forlani et al., 2010). Em termos de tolerância a modificações no ambiente, as espécies exclusivas desse grupo podem ser classificadas como generalistas de áreas abertas (Forlani et al., 2010). As espécies presentes no grupo 3 também podem ser classificadas como generalistas de áreas abertas, porém possuem menor tamanho (variando de espécies com CRC de 29$45 \mathrm{~mm}$ ) e utilizam plantas de menor porte para vocalizar. Essa estruturação das comunidades de anuros ao longo dos sítios de vocalização em função do tamanho dos indivíduos, e pela presença de estruturas morfológicas (por exemplo, discos adesivos), segue o padrão descrito na literatura (BERTOLUCI \& RODRIGUES, 2002). A presença de discos adesivos permite aos hilídeos usar estratos verticais para estabelecimento de sítios de vocalização e com isso habilidade de ocupar maior quantidade de estratos espaciais, se comparado às espécies terrícolas (Pombal JR, 1997).

As espécies de anuros com menor tolerância a modificações no ambiente podem ser afetadas por alterações na paisagem. Dentre os efeitos dessas alterações está o desaparecimento de especialistas em favor de generalistas (VAN Rooy \& STUMPEL, 1995). Corroborando essa teoria, a espécie estritamente arborícola $P$. rohdei foi encontrada somente na poça 3 , onde há um corredor florestal conectando-a ao fragmento de mata.

Ambientes com alta heterogeneidade espacial podem acomodar maior número de espécies (CAMPOS \& VAZ-SILVA, 2010), por apresentarem grande quantidade de sítios de vocalização. Entretando, a presença de corredor florestal conectando os fragmentos florestais aos corpos d'água parece ser o fator primário influenciando a composição e riqueza de anuros, conforme demonstrado pelos resultados dos "clusters". Isso porque as espécies, mesmo sendo capazes de utilizar os sítios de vocalização presentes nos corpos d'água, poderiam ser impedidas de alcançarem os mesmos caso os corredores florestais não estivessem presentes, devido ao baixo grau de permeabilidade da matriz. 
Trabalhos anteriores têm demonstrado que a capacidade de dispersão através da matriz é um dos fatores determinantes na persistência de espécies em áreas fragmentadas (GASCON, 1991; LAURANCE, 1991; Laurance \& BierRegard, 1997). Recentemente, "desconexão do habitat" foi identificada como um mecanismo distinto que influencia na redução da riqueza e abundância de anfíbios de ambientes lóticos em fragmentos florestais na Mata Atlântica (BECKER et al., 2010). A menor riqueza observada nas poças ausentes de corredores florestais pode ser um indício de que a "desconexão do habitat" com consequente perda de microhabitats pode também ocorrer em espécies que se reproduzem em ambientes lênticos.

Contrariamente à nossa expectativa, não houve correlação positiva das variáveis ambientais com a riqueza de espécies em atividade reprodutiva. Apesar disso, temperaturas baixas talvez seja um fator limitante na reprodução de anuros em regiões serranas da Mata Atlântica e podem indicar o término da estação chuvosa (Eterovick \& Sazima, 2000). É visível a preferência de espécies dessa região por períodos chuvosos, os quais coincidem com o aumento das temperaturas. Estudos recentes apontaram que a temperatura máxima está relacionada ao fotoperíodo, que parece ser o fator fenológico fundamental na ativação de processos fisiológicos ligados à reprodução (HATANO et al., 2002; Both et al., 2008; CANavero \& Arim, 2009). Apesar de o fator temporal ter importância variada em outros estudos na região tropical (POMBAL JR, 1997; BERTOLUCI, 1998), estudos de longo prazo na região de estudo são necessários para conseguir mensurar os efeitos de variações anuais.

$\mathrm{Na}$ escala que o presente estudo se propõe analisar, a (des)conexão com os fragmentos florestais pode ser o principal fator que atua na estruturação espacial das comunidades de anuros que utilizam poças para reprodução nessa paisagem fragmentada. Estudos adicionais podem acrescentar informação sobre a influência de diferentes tipos de matrizes no deslocamento dos anfíbios em direção aos corpos d'água.

Agradecimentos. Agradecemos aos colegas Karen H. Beard, Paulo S. Bernarde e Renato Neves Feio pela revisão e considerações no manuscrito. A dois revisores autônomos, pelas sugestões. Ao Sr. Amarildo Uliana e Jaime Stein por permitir nossa entrada na área de estudo e ao Sr. Lourenço M. Júnior e Lamara P. Barbosa pelo auxílio logístico. A Marlene Hoffman pela organização do material biológico e por sua dedicação à coleção zoológica do Museu de Biologia Mello Leitão. A Rogério L. Teixeira pelos ensinamentos e auxílio nos primeiros campos. JFRT foi financiado pela CAPES e pelo National Science Foundation (NSF) (Proc. DEB-1144692 a Rafael O. de Sá.

\section{REFERÊNCIAS BIBLIOGRÁFICAS}

Almeida, A. P.; Gasparini, J. L. \& Peloso, P. L. V. 2011. Frogs of the state of Espírito Santo, southeastern Brazil - The need for looking at the 'coldspots'. Check List 7(4):542-560.

BABbit, K. J. \& TANNER, G. W. 2000. Use of temporary wetlands by anurans in a hydrologically modified landscape. Wetlands
20(2):313-322

Becker, C. G.; Fonseca, C. R.; Haddad, C. F. B. \& Prado, P. I. 2010. Habitat Split as a Cause of Local Population Declines of Amphibians with Aquatic Larvae. Conservation Biology 24(1):287-294.

BernaRde, P. S. \& Anjos, L. 1999. Distribuição espacial e temporal da anurofauna no Parque Estadual Mata do Godoy, Londrina, Paraná, Brasil. Comunicação do Museu de Ciências Tecnológicas 2(1):127-140.

Bertoluci, J. 1998. Annual patterns of breeding activity in Atlantic Rainforest anurans. Journal of Herpetology 32:607-611.

Bertoluci, J. \& Rodrigues, M. T. 2002. Utilização de habitats reprodutivos e micro habitats de vocalização em uma taxocenose de anuros (AMPHIBIA) da Mata Atlântica do Sudeste do Brasil. Papéis Avulsos de Zoologia 42(11):287-297.

Both, C.; Kaefer, I. L.; Santos, T. G. \& Cechin, S. T. Z. 2008. An austral anuran assemblage in the Neotropics: seasonal occurrence correlated with photoperiod. Journal of Natural History 42:205222.

CAmpos, F. S. \& VAZ-SiLVA, W. 2010. Distribuição espacial e temporal da anurofauna em diferentes ambientes no município de Hidrolândia, GO, Brasil Central. Neotropical Biology and Conservation 5(3):179-187.

Canavero, A. \& Arim, M. 2009. Clues supporting photoperiod as the main determinant of seasonal variation in amphibian activity. Journal of Natural History 43:2975-2984.

Casal, F. C. \& Juncá, F. A. 2008. Girino e canto de anúncio de Hypsiboas crepitans (Amphibia: Anura: Hylidae) do estado da Bahia, Brasil, e considerações taxonômicas. Boletim do Museu Paranaense Emílio Goeldi 3(3):217-224.

Chazdon, R. L.; Colwell, R. K.; Denslow, J. S. \& Guariguata, M. R. 1998. Statistical Methods for Estimating Species Richness of Woody Regeneration in Primary and Secondary Rain Forests of NE Costa Rica. In: Dallmeier, F. \& Comiskey, J. A. eds. Forest Biodiversity Research, Monitoring and Modeling: Conceptual Background and Old World Case Studies. Paris, Parthenon Publishing. p.285-309.

Clarke, K. R. 1993. Non-parametric multivariate analyses of changes in community structure. Australian Journal of Ecology 18(1):117-143

Clarke, K. R. \& Gorley, R. N. 2006. PRIMER v6: User Manual/ Tutorial. PRIMER-E, Plymouth. 110p.

Duellman, W. E. 1999. Distribution patterns of amphibians in South America. In: Duellman, W. E. eds. Patterns of distribution of amphibians - a global perspective. Baltimore, The Johns Hopkins University Press. p. 255-328.

Eterovick, P. C. \& Sazima, I. 2000. Struture of an Anuran community in a montane Meadow in Southeastern Brasil: Effects of seasonality, Habitat, and Predation. Amphibia-Reptilia 21(1):439-461.

Feitoza, L. R. 1986. Carta agroclimática do Espírito Santo. Governo do Estado do Espírito Santo: Secretaria de Estado da Agricultura \& Empresa Capixaba de Pesquisa Agropecuária - EMCAPA.

Ferreira, R. B. \& Mendes, S. L. 2010. Herpetofauna no campus da Universidade Federal do Espírito Santo, área urbana de Vitória, Brasil. Sitientibus 10(2-4):279-285.

Ferreira, R. B. \& VRcibradic, D. 2005. Notes on geographic distribution to Hyla polytaenia (Anura, Hylidae). Herpetological Review 36(3):332-333.

Ferreira, R. B.; Silva-Soares, T. \& Rödder, D. 2010. Amphibians of Vitória, an urban area in south-eastern Brazil: first approximation. Salamandra 46(4):187-196.

Forlani, M. C.; Bernardo, P. H.; Haddad, C. F. B. \& Zaher, H. 2010. Herpetofauna do Parque Estadual Carlos Botelho, São Paulo, Brasil. Biota Neotropica 10(3): http://www.biotaneotropica.org. br/v10n3/en/abstract?inventory+bn00210032010.

GASCON, C. 1991. Population and community-level analyses of species occurrences of central amazonian rainforest tadpoles. Ecology 72(5):1731-1746.

Gasparini, J. L.; Almeida, A. P.; Cruz, C. A. G. \& Feio, R. N. 2007. Os anfíbios ameaçados de extinção no estado do Espírito Santo. In: Passamani, M. \& Mendes, S. L. org. Espécies da fauna ameaçadas de extinção no Estado do Espírito Santo. Vitória, Instituto de Pesquisas da Mata Atlântica. p. 75-86.

Hatano, F. H.; Rocha, C. F. D. \& Van Sluys, M. 2002. Environmental factors affecting calling activity of a tropical diurnal frog (Hylodes phyllodes: Leptodactylidae). Journal of Herpetology 36:314-318. 
Hod, W. 1977. Call differences and calling site segregation in anuran species from Central Amazonian floating meadows. Oceologia 28(4):351-363.

INCAPER - Instituto Capixaba de Pesquisa, Assistência Técnica e Extensão Rural. 2006. Sistema de Informação Agrometeorológica/ série histórica/ INMET. Disponível em: <http://www.incaper.es.gov. $\mathrm{br} / \mathrm{clima} / \mathrm{htm}>$. Acesso em: 14.01.2006.

Izeckson, E. \& Carvalho-e-Silva, S. P. 2001. Anfíbios do Município do Rio de Janeiro. Rio de Janeiro, Editora UFRJ. 148p.

KöPPEN, W. 1936. Das geographische System der Klimate. In: KopPES, W. \& R. Geiger, R. eds. Handbuch der Klimatologie. Berlin, Borntrager. p.1-44.

LAURANCE, W. F. 1991. Ecological correlates of extinction proneness in Australian tropical rain forest mammals. Conservation Biology 5(1):79-89.

Laurance, W. F. \& Bierregaard JR, R. O. 1997. Tropical Forest Remnant. Ecology, management, and conservation of fragmented communities. Chicago, University Chicago Press. 616p.

Montesinos, R.; Peloso, P. L. V.; Koski, D. A.; Valadares, A. P. \& Gasparini, J. L. 2012. Frogs and toads of the Pedra Azul Forno Grande Biodiversity Corridor, southeastern Brazil. Check List 8: $102-111$.

Napoli, M. F. 2005. A new species allied to taxa Hyla circumdata (Anura: Hylidae) from Serra da Mantiqueira, Southeastern Brazil. Herpetologica 61(1):63-69.

Pombal JR, J. P. 1997. Distribuição espacial e temporal de anuros (Amphibia) em uma poça permanente na Serra de Paranapiacaba Sudeste do Brasil. Revista Brasileira de Biologia 57(4):583-594.

Prado, G. M. \& Pombal JR, J. P. 2005. Distribuição espacial e temporal dos anuros em um brejo da Reserva Biológica de Duas Bocas, sudeste do Brasil. Arquivos do Museu Nacional 63(4):685-705.

Ramos, A. D. \& Gasparini, J. L. 2004. Anfíbios do Goiapaba-Açu, Fundão, Espírito Santo. Vitória, Gráfica Santo Antônio. 75p.
Rödder, D.; Teixeira, R. L.; Ferreira, R. B.; Dantas, R. B.; Pertel, W. \& Guarneire, G. J. 2007. Anuran hotspots: the municipality of Santa Teresa, southeastern Brazil. Salamandra 43(2):91110 .

Silva-Soares, T.; Ferreira, R. B.; Salles, R. O. L. \& Rocha, C. F D. 2011. Continental, insular and coastal marine reptiles from the municpality of Vitória, state of Espírito Santo, southeastern Brazil. Check List 7(3):290-298.

Teixeira, R. L.; Ferreira, R. B. \& Rödder, D. 2008. Diversity and abundance variations of anurans at a permanent pond in Suruaca's Valley, Linhares, Espirito Santo, southeastern Brazil. Amphibia 7(1):20-25.

Tonini, J. F. R.; Carão, L. M.; Pinto, I. S.; Gasparini, J. L.; Leite, Y. L. R. \& Costa, L. P. 2010. Non-volant tetrapods from Reserva Biológica de Duas Bocas, State of Espírito Santo, Southeastern Brazil. Biota Neotropica 10(3): http://www.biotaneotropica.org. br/v10n3/en/abstract?inventory+bn02710032010.

Tonini, J. F. R.; MendonçA, I. S.; Coutinho, A. B. \& Gasparini, J. L. 2011. Anurans from Costa Bela, state of Espírito Santo, southeastern Brazil: inventory at an urban area and the rediscovery of Allobates in the state. Herpetology Notes 4:435-444.

Van Rooy, P. T. J. \& Stumpel, A. H. P. 1995. Ecological impact of ecomomic development on sardinian herpetofauna. Conservation Biology 9(2):263-269.

Vrcibradic, D.; Ferreira, R. B. \& Teixeira, R. L. 2005. Notes on geographic distribution to Phyllomedusa rohdei. Herpetological Review 21(1):22.

Wogel, H.; Abrunhosa, P. A. \& Pombal, J. P. 2005. Breeding behaviour and mating success of Phyllomedusa rohdei (Anura Hylidae) in south-eastern Brazil. Journal of Natural History 39(22):2035-2045.

ZAR, J. H. 1999. Biostatistical Analysis. 4ed. New Jersey, Prentice Hall. 663p

Recebido em 18 de outubro de 2011. Aceito em 18 de junho de 2012. ISSN 0073-4721

Artigo disponível em: www.scielo.br/isz 